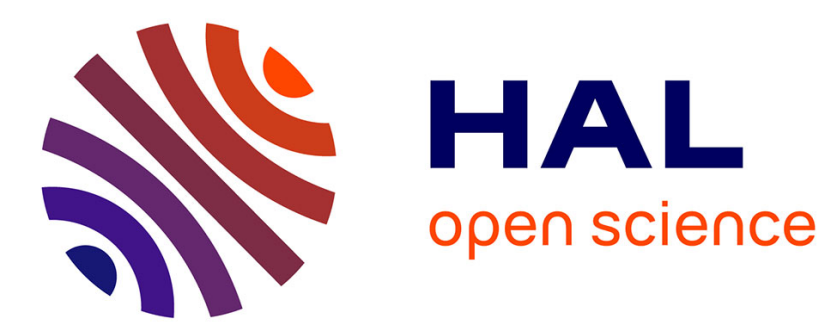

\title{
Fusion of fuzzy spatial relations
}

Nadeem Salamat, El-Hadi Zahzah

\section{- To cite this version:}

Nadeem Salamat, El-Hadi Zahzah. Fusion of fuzzy spatial relations. 2010. hal-00472795

\section{HAL Id: hal-00472795 \\ https://hal.science/hal-00472795}

Preprint submitted on 13 Apr 2010

HAL is a multi-disciplinary open access archive for the deposit and dissemination of scientific research documents, whether they are published or not. The documents may come from teaching and research institutions in France or abroad, or from public or private research centers.
L'archive ouverte pluridisciplinaire HAL, est destinée au dépôt et à la diffusion de documents scientifiques de niveau recherche, publiés ou non, émanant des établissements d'enseignement et de recherche français ou étrangers, des laboratoires publics ou privés. 


\title{
Fusion of fuzzy spatial relations
}

\author{
Nadeem SALAMAT, El-hadi ZAHZAH \\ Université de La Rochelle \\ Laboratoire de Mathématiques, Images et Applications \\ Avenue M Crépeau La Rochelle 17042, France \\ \{nsalamat, ezahzah\}@univ-lr.fr
}

\begin{abstract}
Spatial relations are essential for understanding the image configuration and modeling common sense knowledge. In most of existing methods, topological, directional and distance spatial relations are computed separately as they have separate application domains. Introduction of Temporal Geographic Information System (TGIS), spatiotemporal reasoning and study of spatio-temporal relations required the computation of topological and metric spatial relations together.

In this paper the fuzzy topological and directional relations are integrated with the help of fuzzy Allen relations and directions are evaluated by specific fuzzy membership functions. A matrix of fuzzy relations is developed where the topological and directional relations are integrated for a $2 \mathrm{D}$ scene. Experiments are performed to validate the proposed method. The results are analyzed and interpreted from histograms.
\end{abstract}

Keywords: Topological and metric relations, Fuzzy topological relations, Fusion of spatial relations, Fuzzy directional relations, Matrix of fuzzy relations.

\section{Introduction}

Temporal Geographic Information System (TGIS) was introduced in recent years. This enhancement of Geographic Information System (GIS) deeply affects the present studies in GIS and need fusion of different information provided from different sensors given heterogenous data. The fusion of spatial relations will be helpful in defining the spatio-temporal relations, reasoning in many decision processus. In existing methods, topological, directional and distance spatial relations are studied separately. Beside this information, fuzzy spatial relations are also important for fuzzy reasoning, fuzzy image understanding and fuzzy change detection. Fuzzy topological relations are studied by the extended models of 9 -intersections [5,4] and Region Connected Calculus (RCC8) [13, 14, 16]. These topological relations are studied through the object model with intermediate boundaries or the well known Egg-Yolk model [2,3]. Fuzzy directional relations can be defined using the mathematical morphology as in [7] and numerical methods as in $[11,10]$. Fuzzy directional relations assign a degree to each cardinal direction and different methods are used to assign the degree to a cardinal direction. Another type of metric relation is the distance relations. 
These relations provide us the distance information about the closet part of objects and a fuzzy membership function assigns a degree to the distance relation Near, Far and Far off [1]. In the approaches for finding the fuzzy directional relations, crisp objects are considered and fuzzy objects are treated by considering the Egg's boundary. Fuzzy methods for directional relations work for the limited set of topological relations. All sort of relations are essential for image understanding, modeling common sense knowledge, natural phenomenon and spatial reasoning [6]. This requirement of image understanding needs to apply different methods to find the topological, directional and distance relations between the objects and it is needed to have a single method where we can get all the required information about the spatial scene. In this work two types of fuzzy sets are used, $1 D$ fuzzy sets for finding the fuzzy Allen relations and then $1 D$ directional fuzzy sets for evaluating the directions in $2 D$ scene. This method of using the $1 D$ fuzzy sets provide us the integrated topological and directional information of a $2 D$ scene. These integrated information of a $2 D$ scene are represented in a matrix. Each entity of this matrix represents the percentage surface area of two objects having a topological relation in a specific direction.

This paper is structured as follows, section 2 discusses in detail the different terms and computations necessary for $1 D$ Allen relations, in section 3 the fusion of topological and directional relations and their interpretation is given. Results for different configurations is given in section 4 . Section 5 concludes the paper.

\section{Terminology used for computation fuzzy Allen relations}

\subsection{Oriented lines, segments and longitudinal sections}

Let $A$ and $B$ be two spatial objects and $(v, \theta) \in \mathbb{R}$, where $v$ is any real number and $\theta \in[0,2 \pi] . \Delta_{\theta}(v)$ is an oriented line at orientation angle $\theta . A \cap \Delta_{\theta}(v)$ is the intersection of object $A$ and oriented line $\Delta_{\theta}(v)$. It is denoted by $A_{\theta}(v)$, called segment of object $A$ and its length is $x$. Similarly for object $B$ where $B \cap \Delta_{\theta}(v)=B_{\theta}(v)$ is segment and $z$ is its length. $y$ is the difference between the maximum value of $B \cap \Delta_{\theta}(v)$ and minimum of $A \cap \Delta_{\theta}(v)$ (for details [12]). In case of polygonal object approximations $(x, y, z)$ can be calculated from intersecting points of line and object boundary. If there exist more than one segment, then it is called longitudinal section.In this paper all the 180 directions are considered with an angle increment of one degree and lines are drawn by $2 D$ Bresenham digital line algorithm. A polygonal object approximation is taken and lines passing through the polygon vertices are taken into account.

\section{$2.21 D$ Allen relations in space}

Allen [8] relations are arranged as $A=\left\{<, m, o, s, f, d, e q, d_{i}, f_{i}, s_{i}, o_{i}, m_{i},>\right\}$ with meanings before, meet, overlap, start, finish, during, equal, during_by, fin$i s h \_b y$, start_by, overlap_by, meet_by, and after. All the Allen relations in space are conceptually illustrated in figure 1. These relations have a rich support for the topological and directional relations. In the neighborhood graph of 


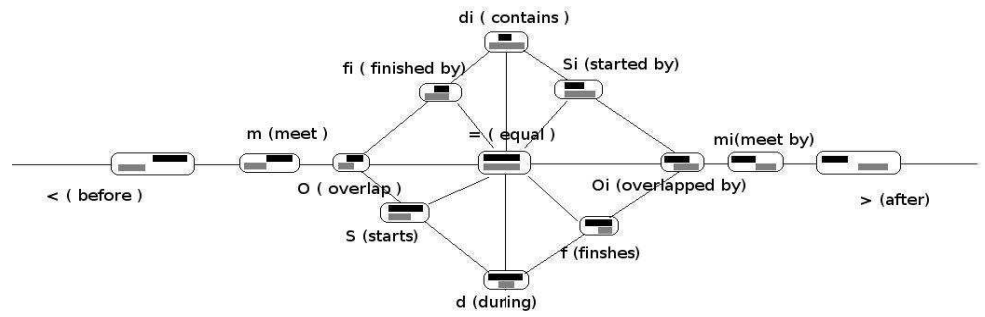

Fig. 1. Black segment represents the reference object and gray segment represents argument object

Allen relations, three paths can be find (because we assume that objects are monolithic and don't changing size during their movement. Some possible paths are ignored due to this reason ). Depending upon the neighborhood graph of Allen relations, Inverse of these relations can be divided into two categories, object commutativity and reorientation of relations. According to reorientation of a relation, we can write $A_{1}=\left\{<, m, o, S, d, f_{i}, d_{i},=\right\}$ and their inverses as $A_{2}=\left\{>, m_{i}, o_{i}, f, d, S_{i}, d_{i},=\right\}$. This shows that relations $d,=, d_{i}$ have their own reorientations. Region Connected Calculus - RCC8 relations are possible combination of 8 independent Allen relations in $1 D$. These relations and their inverse show that the whole $2 D$ space can be explored with the help of $1 D$ Allen relations using oriented lines varying from $(0, \pi)$.

\section{$2.3 \quad$ Fuzzification of Allen relations}

Fuzzification process of Allen relations do not depend upon particular choice of fuzzy membership function, trapezoidal membership function is used due to flexibility in shape change. Let $r(I, J)$ be Allen relation between segments $I$ and $J$ where $I \in A$ (argument object) and $J \in B$ (reference object), $r^{\prime}$ is the distance between $r(I, J)$ and its conceptional neighborhood. We consider a fuzzy membership function $\mu: r^{\prime} \longrightarrow[0,1]$. The fuzzy Allen relations defined by Matsakis [12] are

$-f_{<}(I, J)=\mu_{(-\infty,-\infty,-b-3 a / 2,-b-a)}(y)$,

$-f_{>}(I, J)=\mu_{(0, a / 2, \infty, \infty)}(y)$

- $f_{m}(I, J)=\mu_{(-b-3 a / 2,-b-a,-b-a,-b-a / 2)}(y)$,

- $f_{m i}(I, J)=\mu_{(-a / 2,0,0, a / 2)}(y)$

- $f_{O i}(I, J)=\mu_{(-a,-a / 2,-a / 2,0)}(y)$

- $f_{s i}(I, J)=\min \left(\mu_{(-(b+a) / 2,-a,-a,+\infty)}(y), \mu_{(-3 a / 2,-a,-a,-a / 2)}(y), \mu_{(z, 2 z,+\infty,+\infty)}(x)\right)$

- $f_{d}(I, J)=\min \left(\mu_{(-b,-b+a / 2,-3 a / 2,-a)}(y), \mu_{(-\infty,-\infty, z / 2, z)}(x)\right)$

where $a=\min (x, z), b=\max (x, z)$ and $x$ is the length of longitudinal section of argument object $A$ and $z$ is the length of longitudinal section of reference object $B$. Most of relations are defined by one membership function like be fore, after,meet, meet_by and some of them are defined by more than one membership functions like $d($ during $), d_{i}($ during_by $), f$ (finish),$f_{i}$ (finished_by). In 
fuzzy set theory, sum of all the relations is one, this gives the definition for relation fuzzy equal. During the decomposition process of an object into segments, there can be multiple segments depending on object shape and boundary which is called longitudinal section. Different segments of a longitudinal section are at a certain distance and these distances might effect end results. In polygonal object approximation, fuzzy T-conorms are used for fuzzy integration of available information. Here for simplicity only T-conorm (Fuzzy $O R$ operator)is used.

$$
\mu_{(O R)}(u)=\max \left(\mu_{(A)}(u), \mu_{(B)}(u)\right)
$$

\subsection{Normalized fuzzy histogram of Allen relations}

Histogram of fuzzy Allen relation represents total area of subregions of $A$ and $B$ that are facing each other in given direction $\theta$. Mathematically it can be written as [15]

$$
\int_{-\infty}^{+\infty}\left(\sum_{r \in A} F_{r}\left(\theta, A_{\theta}(v), B_{\theta}(v)\right) d v=(x+z) \sum_{k=1}^{n} r\left(I_{k}, J_{k}\right)\right.
$$

where $z$ is the area of reference object and $x$ is area of augmented object in direction $\theta, n$ is total number of segments treated and $r\left(I_{k}, J_{k}\right)$ is an Allen relation for segments $\left(I_{k}, J_{k}\right)$. These histograms are normalized by dividing all Allen's relations by their sum for every $\theta$. It is represented by $\left\lceil F_{r}^{A B}(\theta)\right\rfloor$ where $r \in$ $A$. $\left\lceil F_{r}^{A B}(\theta)\right\rfloor=\frac{F_{r}^{A B}(\theta)}{\sum_{\rho \in A} F_{\rho}^{A B}(\theta)}$. These fuzzy Allen relations are directional fuzzy numbers and can be used to define the quantitative fuzzy directional relations.

\section{Fusion of Topological and directional relations}

This section consists of two subsections where in first one it is described how different functions can be used to assess the fuzzy qualitative directions and how the different Allen relations are combined for topological and directional information fusion. In second subsection, the representation method for the fuzzy topological and fuzzy directional relations is described in detail.

\subsection{Fusion of topological and directional Components}

All these equations depicted in section 2.3 assign a numerical value to a topological spatial relation in a direction $\theta$. To find directional contents of a topological relation, these numerical values are used with directional fuzzy sets. Directions are represented as $\{E, N E, N, N W, W, S W, S, S E\}$ with meanings East, North_East, North, North_West, West, South_West, South and South_East. To assess these fuzzy directional relations, two trigonometric membership functions $\cos 2 \theta$ and $\sin 2 \theta$ are used. As angle distribution is taken to the half plane so opposite Allen relations are used to define the opposite directions except the direction East and West where union of both relations are used. Mathematically these relations can be defined as 


$$
\begin{aligned}
& -f_{E}=\sum_{\theta=0}^{\frac{\pi}{4}} A_{r_{2}} \times \cos ^{2}(2 \theta)+\sum_{\theta=\frac{3 \pi}{4}}^{\pi} A_{r_{1}} \times \cos ^{2}(2 \theta) \\
& -f_{W}=\sum_{\theta=0}^{\frac{\pi}{4}} A_{r_{1}} \times \cos ^{2}(2 \theta)+\sum_{\theta=\frac{3 \pi}{4}}^{\pi} A_{r_{2}} \times \cos ^{2}(2 \theta) \\
& -f_{N}=\sum_{\theta=\frac{\pi}{4}}^{\frac{3 \pi}{4}} A_{r_{2}} \times \cos ^{2}(2 \theta) \\
& -f_{S}=\sum_{\theta=\frac{\pi}{4}}^{\frac{3 \pi}{4}} A_{r_{1}} \times \cos ^{2}(2 \theta)
\end{aligned}
$$

Where $A_{r_{i}} \in A_{i}, i=1,2$ given in section 2.2 and $f \in\{D, E C, P O, T P P, N T P P, T P P I, N T P P I, E Q\}$ which respectively mean Disjoint, Externally connected, Partially overlap, Tangent properpart,

Non tangent properpart, Tangent properpart inverse, Non Tangent properpart inverse, and Equal.

\subsection{Interpretation of topological and directional relations}

The relations are manipulated in $(8 \times 8)$ matrix where $C(i, j)$ represents the $i^{\text {th }}$ topological relation in $j^{t h}$ direction. Rows are arranged in an order of $\{D, E C, P O, T P P, N T P P, T P P I, N T P P I, E Q\}$. Different directions may yield different $1 D$ relations in $2 D$ space and different relations may coexist along the same direction. Below a conclusion about the overall $2 D$ relation is given.

1. Only first row is non zero then objects have fuzzy disjoint $(D C)$ topological relation .

2. If the first and the second rows are non zero then the overall relation in $2 D$ space is fuzzy meet $E C$.

3. If there exist at least one non zero value in third row it means there exist fuzzy topological relation overlap. or If non zero values also exist in TPP along with NTPP (TPPI along with NTPPI) then the relation will be TPP $(N T P P)$ in the corresponding direction.

4. Relations $P P, P P I, E Q$ hold if the corresponding relation holds in all directions. A relation will hold if all elements in a row are non zero and all other rows are zero.

The above explanation shows that overlap relation in $2 D$ space is more complex than any other topological relation. In this case all the Allen relations coexist in different directions.

\section{Experiments and discussion}

In the following set of examples the objects $A$ (argument object) and $B$ (reference object) are represented by light and dark grey colors respectively. A matrix of fuzzy topological and directional relations are computed. Figure (2(a)) shows that argument object $A$ meets the reference object $B$ in direction East, their topological and directional relations show that there exist a topological relation meet $E C$ with a maximum degree in direction East along with other neighboring directions and Disjoint topological relation exists due to application of 


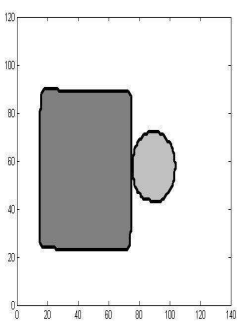

(a)

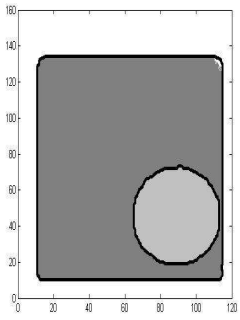

(e)

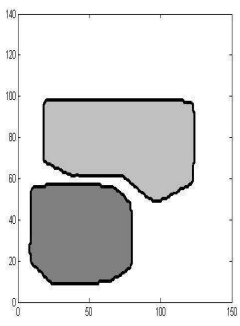

(i)

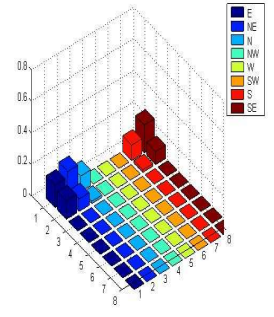

(b)

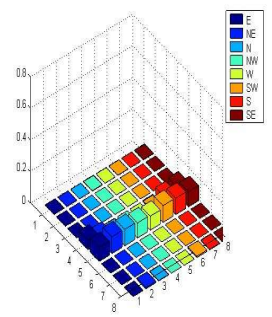

(f)

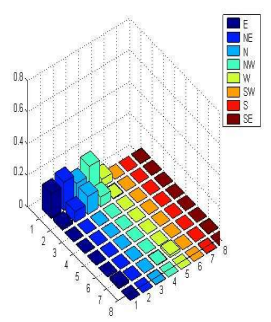

(j)

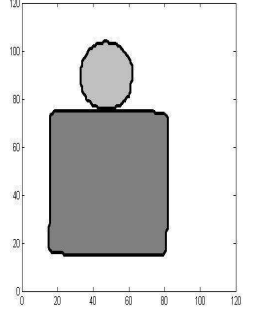

(c)

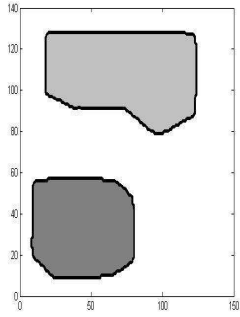

(g)

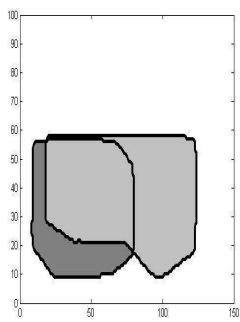

$(\mathrm{k})$

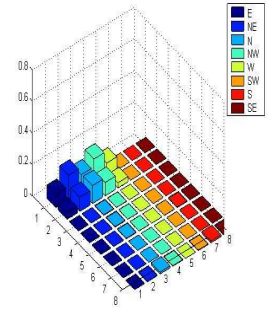

(d)

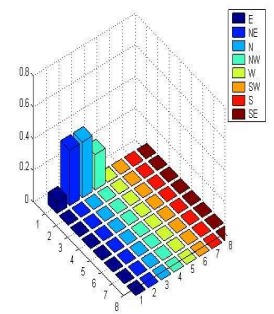

(h)

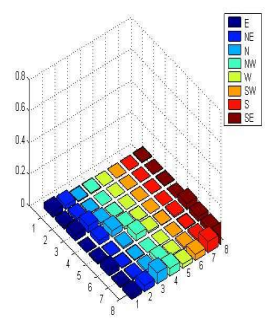

(l)

Fig. 2. Object pairs with meet topological relation and their directional relations

$1 D$ Allen relations (figure 2(b)). Argument object $A$ in figure 2(c) touches the reference object from North, their relation matrix validates the said relation (2(d)). In figure 2(e) argument object lies inside the reference object near the boundary in the East and South_East direction, their relations matrix (figure $2(\mathrm{f})$ ) shows that the TPP topological relation exists with equal degree in the East and South_East direction. In figure 2(g), objects are disjoint and fuzzy relations matrix shows that object lies in North_East, North, North_West to the reference object. Directional components as shown in figure $2(\mathrm{~h})$ specify the object location. This shows that object equally lies in North_East and North directions. In figures 2(i), fuzzy topological relation $E C$ exits due to fuzziness of Allen relations. Disjoint relation exist in directions $E, N E, N, N W$ and $W$ with different degree due to fuzziness of directions. In figure $2(\mathrm{k})$ objects overlap and 
a topological relation $P O$ exists but existence of other relations like $D, E C$ represented in figure $2(\mathrm{j})$.

In the last example, the method is applied to a well known sequence of images "water surface sequence" [9] where a manual segmentation of objects is performed. The tree is taken as a reference object $B$ while the shape of the person is taken as an argument object $A$. In figure $3(\mathrm{a})$ as the argument (person) object is very close to the reference object (tree). due to this closeness, there exist fuzzy meet (EC) relation in a certain directions along with the fuzzy disjoint relation in a specified directions. There exist number of directions because the object is very close and visual rang is too much large (results are shown in figure 3(b)). As the argument object walks away from the reference object (3(c)), their topological relations also change. Now only fuzzy disjoint topological relation exists and due to the distance between the objects, their visual rang also decreases and only $E, N E, S E$ directional relations exist (figure $3(\mathrm{~d})$ ). In all the above exam-

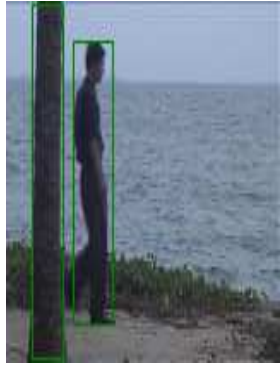

(a) Frame 1499

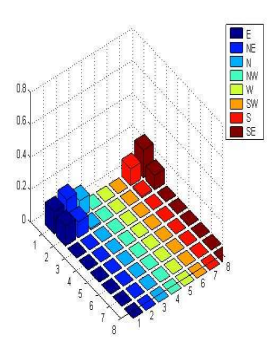

(b) Spatial relations

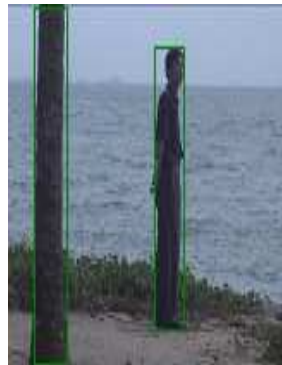

(c) Frame 1547

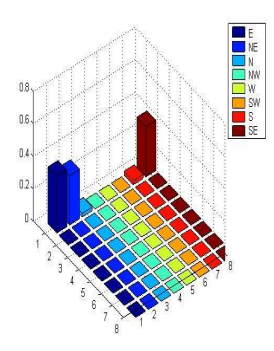

(d) Spatial relations

Fig. 3. Difference frames of image sequence "watersurface" and their spatial relations.

ples, it is shown that the topological and directional relations can be successfully integrated in a single method. This method will be hopefully used for spatiotemporal reasoning where we need the topological and directional information at each step.

\section{Conclusion}

Spatial topological and directional relations are important to understand the scene configuration and spatio-temporal reasoning. Spatio-temporal information fusion is important to have a single method to understand the image configuration completely. In this paper, the key point is that different informations regarding the topological and directional relations are integrated through a single method. These relations are sensitive to the distance between the objects and cardinal directions. Due to the spatio-temporal nature it is possible to construct from the fuzzy Allen relations a set of spatio-temporal relations such as Leave,Enter, Cross, Bypass and others. This method could be used for spatio- 
temporal reasoning. Our future work will now in defining the spatio-temporal relations of the above mentioned classes between two moving spatial objects.

\section{References}

1. Bloch, I., Maitre, H.: Fuzzy distances and image processing. In: SAC '95: Proceedings of the 1995 ACM symposium on Applied computing. pp. 570-574. ACM, New York, NY, USA (1995)

2. Clementini, E., Felice, P.D.: An algebraic model for spatial objects with indeterminate boundaries. In: In P. Burrough A. Frank, Geographic objects with indeterminate. pp. 155-169. Taylor and Francis (1996)

3. Cohn, A.G., Gotts, N.M.: The 'egg-yolk' representation of regions with indeterminate boundaries. In: Burrough, P., Frank, A.M. (eds.) Proceedings, GISDATA Specialist Meeting on Geographical Objects with Undetermined Boundaries. pp. 171-187. Francis Taylor (1996)

4. Du, S., Qin, Q., Wang, Q., Li, B.: Fuzzy description of topological relations i: A unified fuzzy 9-intersection model. In: ICNC (3). pp. 1261-1273 (2005)

5. Egenhofer, M.J., Sharma, J., Mark, D.M.: A Critical Comparison of The 4Intersection and 9-Intersection Models for Spatial Relations: Formal Analysis. Auto-Carto 11 pp. 1-12 (1993)

6. Hudelot, C., Atif, J., Bloch, I.: Fuzzy Spatial Relation Ontology for Image Interpretation. Fuzzy Sets Syst. 159(15), 1929-1951 (2008)

7. Isabelle Bloch, A.R.: Directional Relative Position Between Objects in Image Processing: A Comparison Between Fuzzy Approaches. patter Recognition 36, 1563$1582(2003)$

8. JF, A.: Maintaining Knowledge about Temporal Intervals . Communications of the ACM 26(11), 832-843 (1983)

9. Li, L., Huang, W., Gu, I.Y.H., Tian, Q.: Statistical modeling of complex backgrounds for foreground object detection. IEEE Transactions on Image Processing 13(11), 1459-1472 (2004)

10. Matsakis, P., Keller, J.M., Wendling, L.: F-Histograms and Fuzzy Directional Spatial Relations. In: Proceedings, LFA' 99 (French-Speaking Conference on Fuzzy Logic and Its Applications. pp. 207-213 (1999)

11. Miyajima, K., Ralescu, A.: Spatial Organization in 2D Segmented Images: Representation and Recognition of Primitive Spatial Relations. Fuzzy Sets Syst. 65(2-3), 225-236 (1994)

12. P.Matsakis, Nikitenko, D.: Combined Extraction of Directional and Topological Relationship Information from 2D Concave Objects, in fuzzy modeling with spatial informations for geographic problems. Springer-Verlag Publications, pp. 15-40, New York (2005)

13. Randell, D., Cui, Z., Cohn, A.: A spatial logic based on regions and connection. In: Proc. 3rd Int. Conf. on Knowledge Representation and Reasoning. pp. 165-176. Morgan Kaufmann, San Mateo (1992)

14. Rosenfeld, A.: Fuzzy digital topology. Information and Control 40(1), 76-87 (1979)

15. Salamat, N., hadi Zahzah, E.: Spatial relations analysis by using fuzzy operators. In: ICCS (2). Lecture Notes in Computer Science, vol. 5545, pp. 395-404. Springer (2009)

16. Tang, X.: Spatial object model[1]ing in fuzzy topological spaces : with applications to land cover change. Ph.D. thesis, Enschede (2004), http://doc.utwente.nl/ $41448 /$ 